\title{
Symbols for physical constants and properties
}

Tab. 1: Symbols for physical constants and properties frequently used in this book.

\begin{tabular}{lll}
\hline Quantity & Symbol & Value or typical units \\
\hline Permittivity of free space & $\varepsilon_{0}$ & $8.854 \times 10^{-12} \mathrm{~F} / \mathrm{m}$ \\
\hline Dielectric constant (relative permittivity) & $\varepsilon_{\mathrm{r}}$ & Dimensionless \\
\hline Unit charge & $e$ & $1.602 \times 10^{-19}$ coulombs \\
\hline Energy of an electronic state & $E$ & $\mathrm{eV}$ \\
\hline Fermi energy & $E_{\mathrm{F}}$ & $\mathrm{eV}$ \\
\hline Band gap energy & $E_{\mathrm{g}}$ & $\mathrm{eV}$ \\
\hline Urbach energy & $E_{\mathrm{U}}$ & $\mathrm{meV}$ \\
\hline Density of states (DOS) & $g(E)$ & $\mathrm{cm}-3$ \\
\hline Reduced Planck constant & $\hbar$ & $6.582 \times 10^{-16} \mathrm{eV} \cdot \mathrm{s}$ \\
\hline Boltzmann constant & $k_{\mathrm{B}}$ & $8.617 \times 10^{-5} \mathrm{eV} / \mathrm{K}$ \\
\hline Mass of a free electron & $m_{\mathrm{e}}$ & $9.109 \times 10^{-31} \mathrm{~kg}$ \\
\hline Effective mass of the charge carrier & $m^{\star}$ & $\mathrm{kg}$ \\
\hline Charge carrier mobility & $\mu$ & $\mathrm{m} /(\mathrm{V} \cdot \mathrm{s})$ \\
\hline Charge carrier concentration & $n_{\mathrm{c}}$ & $\mathrm{cm}$ \\
\hline Sheet resistance & $R_{\mathrm{s}}$ & $\Omega / \mathrm{sq}$ \\
\hline Electrical conductivity & $\sigma$ & $\mathrm{S} / \mathrm{cm}$ \\
\hline Absolute temperature & $T$ & $\mathrm{Kelvin}$ \\
\hline & & \\
\hline
\end{tabular}


